\title{
High-resolution spectroscopic observations of the metal-poor, chemically peculiar, and high velocity Fehrenbach \& Duflot star $\star, \star \star$
}

\author{
N. A. Drake ${ }^{1}$ and C. B. Pereira ${ }^{2}$ \\ 1 Sobolev Astronomical Institute, St. Petersburg State University, Universitetski pr. 28, St. Petersburg 198504, Russia \\ 2 Observatório Nacional, Rua José Cristino, 77, CEP 20921-400, São Cristóvão, Rio de Janeiro-RJ, Brazil \\ e-mail: [drake,claudio]@on.br
}

Received 6 February 2010 / Accepted 5 May 2011

\begin{abstract}
Aims. We determine the atmospheric parameters and abundance pattern of the chemically peculiar metal-poor Fehrenbach \& Duflot (Feh-Duf) star to more clearly understand its evolutionary state and the nature of the s-element enhancement in this star.

Methods. Its atmospheric parameters and chemical abundances were determined using high resolution optical spectroscopy and employing the local-thermodynamic-equilibrium model atmospheres of Kurucz and spectral analysis code MOOG.

Results. The derived abundances show that the Feh-Duf star is a low-metallicity $([\mathrm{Fe} / \mathrm{H}]=-1.93)$ star with high carbon and heavy s-element abundances, while the abundance of the light s-process element yttrium is low $([\mathrm{Y} / \mathrm{Fe}]=-0.07)$. The oxygen abundance is lower than for Galactic halo stars of similar metallicity.We conclude that the Feh-Duf star could be a $\mathrm{CH}$ star with $\mathrm{C} / \mathrm{O}=1.3$. Another possibility is that the Feh-Duf star could be an early-AGB star. The Fehrenbach \& Duflot star is also a lead star with $[\mathrm{Pb} / \mathrm{Ce}]=+0.69$. In addition, it displays an extreme retrograde motion $\left(V_{\mathrm{GRF}}=-259 \mathrm{~km} \mathrm{~s}^{-1}\right)$, which in combination with its underabundance of $\alpha$-elements suggests that this star may have been captured by the Milky Way galaxy.
\end{abstract}

Key words. stars: abundances - stars: individual: Feh-Duf - stars: chemically peculiar - stars: population II stars: kinematics and dynamics - stars: fundamental parameters

\section{Introduction}

The Fehrenbach \& Duflot star (=2MASS J05025386-6544207, hereafter the Feh-Duf star according to SIMBAD designation) was discovered in 1981 by Fehrenbach \& Duflot (1981) during their measurements of radial velocities of stars in the region of the Large Magellanic Cloud. They found that this star has a very high radial velocity $\left(+440 \mathrm{~km} \mathrm{~s}^{-1}\right)$ and also displays exceedingly strong $\mathrm{CH}$ absorption bands in its spectrum. These spectral characteristics are typical of $\mathrm{CH}$ stars, a group of peculiar late-type population II giants whose overabundance of carbon and of s-process elements is explained by mass-transfer in a binary system from a former asymptotic giant branch (AGB) star that is now a white dwarf. In this work, we present a highresolution spectroscopic study of the Feh-Duf star to determine its abundance pattern and discuss its evolutionary state as well as a possible extragalactic origin for this star.

\section{Observations}

The high-resolution spectra of the Feh-Duf star analysed in this work were obtained with the FEROS (Fiberfed Extended Range Optical Spectrograph) echelle spectrograph (Kaufer et al. 1999) at the $2.2 \mathrm{~m}$ ESO telescope at La Silla (Chile) on the night of October 20, 2008. The FEROS spectral resolving power is

\footnotetext{
* Based on observations made with the $2.2 \mathrm{~m}$ telescope at the European Southern Observatory (La Silla, Chile).

$\star \star$ Tables 1 and 3 are available in electronic form at http: //www. aanda.org
}

$R=48000$, corresponding to 2.2 pixels of $15 \mu \mathrm{m}$, and the wavelength coverage goes from $3800 \AA$ to $9200 \AA$. The nominal signal-to-noise ratio (hereafter $S / N$ ) was evaluated by measuring the rms flux fluctuation in selected continuum windows, and the typical value being found to be $S / N=100-150$ after two exposures of $3600 \mathrm{~s}$. The spectra were reduced with the MIDAS pipeline reduction package consisting of the following standard steps: CCD bias correction, flat-fielding, spectrum extraction, wavelength calibration, correction of barycentrer velocity, and spectrum rectification. Figure 1 shows the spectrum of the Feh-Duf star in the 6130-6170 ̊ region. A strong redshift of about $9.2 \AA$ is observed.

\section{Analysis and results}

\subsection{Line selection, measurements, and oscillator strengths}

The atomic absorption lines selected in this study are basically the same as those used in previous studies devoted to the analysis of the photospheric abundances of chemically peculiar stars (Pereira \& Drake 2009). The selected lines are sufficiently unblended to yield reliable abundances. Table 1 shows the Fe I and Fe II lines employed in the analysis, the lower excitation potential $\chi$ of the transitions, the $\log g f$ values, the measured equivalent widths $E W_{\lambda}$ and the derived iron abundances of each line. The latter were obtained by fitting Gaussian profiles to the observed ones. The $\log g f$ values for the $\mathrm{Fe}_{\mathrm{I}}$ and $\mathrm{Fe}$ II lines given in Table 1 were taken from Lambert et al. (1996). 


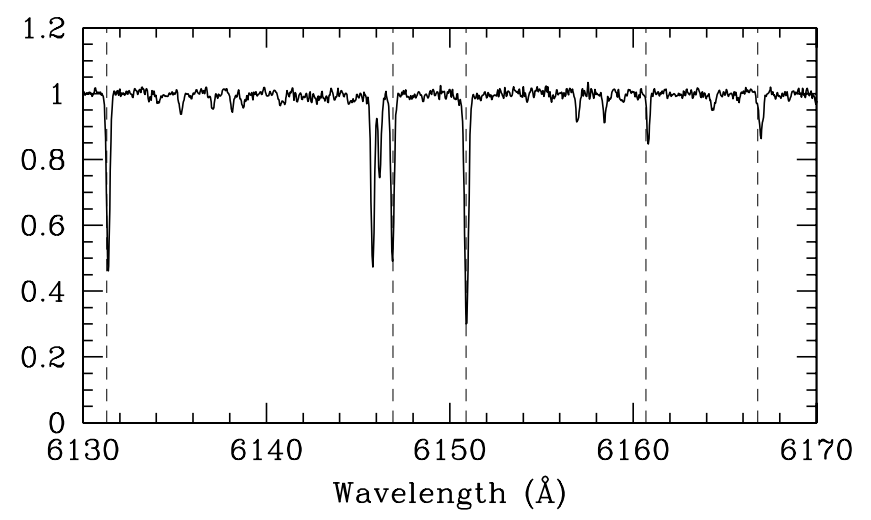

Fig. 1. Spectrum of the Feh-Duf star analyzed in this work. Short dashed vertical lines show the transitions of $\mathrm{Ca}$ I 6122.23, Fe I 6137.70, Ba II 6141.73, Fe I 6151.62, and Fe I 6157.73. The spectral lines are strongly shifted toward the red by about $9.2 \AA$ because of the radial velocity of $448.3 \mathrm{~km} \mathrm{~s}^{-1}$.

Table 2. Atmospheric parameters, radial velocity, and Galactic latitude of Feh-Duf.

\begin{tabular}{lc}
\hline \hline Parameter & Value \\
\hline$T_{\text {eff }}(\mathrm{K})$ & $4500 \pm 120$ \\
$\log g(\mathrm{dex})$ & $0.9 \pm 0.2$ \\
{$[\mathrm{Fe} / \mathrm{H}](\mathrm{dex})$} & $-1.93 \pm 0.10$ \\
$\xi\left(\mathrm{km} \mathrm{s}^{-1}\right)$ & $1.9 \pm 0.3$ \\
$V_{r}\left(\mathrm{~km} \mathrm{~s}^{-1}\right)$ & $+448.3 \pm 0.6$ \\
gal. latitude $\left(b^{\circ}\right)$ & -35.6 \\
\hline
\end{tabular}

\subsection{Determination of the atmospheric parameters}

The determination of atmospheric parameters, effective temperature $\left(T_{\text {eff }}\right)$, surface gravity $(\log g)$, microturbulence $(\xi)$, and metallicity $([\mathrm{Fe} / \mathrm{H}])$ (throughout, we use the notation $[\mathrm{X} / \mathrm{H}]=$ $\left.\log (N(\mathrm{X}) / N(\mathrm{H}))_{\star}-\log (N(\mathrm{X}) / N(\mathrm{H}))_{\odot}\right)$ are prerequisites for abundance determination. To derive these parameters, we followed exactly the procedures described in previous studies of chemically peculiar stars (Drake \& Pereira 2008; Pereira \& Drake 2009). The final derived atmospheric parameters of the Feh-Duf star are given in Table 2.

The internal errors in the adopted effective temperature $\left(T_{\text {eff }}\right)$ and microturbulent velocity $(\xi)$ can be determined from the uncertainty in the slope of the $\mathrm{Fe}_{\mathrm{I}}$ abundance versus excitation potential and $\mathrm{Fe}$ I abundance versus reduced equivalent width $\left(E W_{\lambda} / \lambda\right)$ relations. These quantities are given in Table 2 . The standard deviation in $\log g$ was set by varying this parameter around the adopted solution until the Fe I and Fe II mean abundances differed by exactly one standard deviation of the $[\mathrm{Fe} \mathrm{I} / \mathrm{H}]$ mean value. The value of $\log g$ quoted in Table 2 was determined by means of ionization balance using the assumption of local thermodynamic equilibrium (hearafter LTE). On the other hand, it is well-known that for low-gravity and low-metallicity stars Fe I abundance is subject to significant non-LTE (hereafter NLTE) effects (e.g. Korn et al. 2003; Thevenin \& Idiart 1999, and references therein). However, a consensus of the expected magnitude of NLTE effects has not yet been achieved and a full discussion of NLTE effects is beyond the scope of this paper. Mashonkina et al. (2011) carried out a detailed study of the NLTE mechanisms affecting the formation of neutral and singlyionized iron lines in stellar atmospheres, covering a broad range of effective temperatures, gravities, and metallicities. These authors have developed a comprehensive atomic model for neutral and singly-ionized iron and found that NLTE leads to positive abundance corrections, in agreement with previous studies. However, the magnitude of the corrections that they found is smaller than had been found in earlier results. For example, for the very low metallicity cool giant HD $122563\left(T_{\text {eff }}=\right.$ $4600 \mathrm{~K}, \log g=1.60,[\mathrm{Fe} / \mathrm{H}]=-2.50)$, they found that the average NLTE abundance correction amounts to +0.10 dex for $\mathrm{Fe}$ II lines and +0.19 dex for Fe I lines. Assuming that LTE leads to a discrepancy in the abundances inferred for Fe II and Fe I of about 0.1 dex, we estimated its influence on the surface gravity determination to be +0.25 . In Sect. 3.4, we estimate the effects of this uncertainty in $\log g$ on the derived abundances.

\subsection{Abundance analysis}

The abundances of chemical elements were determined with LTE model-atmosphere techniques. In brief, equivalent widths were calculated by integration through a model atmosphere and were then compared with the observed equivalent widths. This calculation is repeated, changing the abundance of the element in question, until a match is achieved. The LTE atmosphere models of Kurucz (1993) and the current version of the linesynthesis code Moog (Sneden 1973) were used to carry out the calculations.

Table 3 shows the atomic lines used to derive the abundances of the elements as well as the abundances derived for each individual line and Table 4 provides the number of lines employed (or the number of spectral regions in the case of carbon and nitrogen whose abundances were determined using molecular lines) for each species, $n$, the derived abundances with their respective standard deviations, and the $[\mathrm{X} / \mathrm{H}]$ and $[\mathrm{X} / \mathrm{Fe}]$ ratios as well as the $\mathrm{C} / \mathrm{O}$ and the carbon isotopic ${ }^{12} \mathrm{C} /{ }^{13} \mathrm{C}$ ratio.

The abundance of manganese is based on the MnI lines $\lambda 5420$ and $\lambda 6021 \AA$. All fine-structure and hyperfine-structure components for the lines of Mn I were explicitly included. The $g f$-values for the Mn I lines were taken from del Peloso et al. (2005).

The lead abundance was derived from the $\mathrm{Pb}$ I line at 14057.81 A. The line data, which include isotopic shifts and hyperfine splitting, were taken from Van Eck et al. (2003).

Carbon, nitrogen, and oxygen abundances, as well as the ${ }^{12} \mathrm{C} /{ }^{13} \mathrm{C}$ isotopic ratio, were also determined using the spectrum synthesis technique. Since the abundances of the CNO elements are interdependent because of the association of carbon and oxygen in $\mathrm{CO}$ molecules in the atmospheres of cool giants, the CNO abundance determination procedure was iterated until all the abundances of these three elements agreed. The abundances of carbon, nitrogen, and the ${ }^{12} \mathrm{C} /{ }^{13} \mathrm{C}$ isotopic ratio were determined using the lines of the $\mathrm{CH}, \mathrm{CN}$, and $\mathrm{C}_{2}$ molecules. The line lists were assembled by ourselves are similar to those of Drake \& Pereira (2008) and Pereira \& Drake (2009) who studied the chemically peculiar metal-poor stars HD 104340, HD 206983, HD 10613, and BD+04²466. Nevertheless, studying the Fehrenbach \& Duflot star we have updated the value of the dissociation potential of the $\mathrm{CN}$ molecule. In the above mentioned papers, we used the value $D_{0}(\mathrm{CN})=7.65 \mathrm{eV}$ determined by Engleman \& Rouse (1975). More recent studies based on experimental determinations (Huang et al. 1992; Costes et al. 1990) as well as on theoretical calculations (Pradhan et al. 1994), showed that the dissociation energy of the CN molecule is higher, about $7.75 \mathrm{eV}$, and this value was used in the synthetic spectra calculations. 
Table 4. Chemical abundances derived for the Feh-Duf star in the scale $\log \varepsilon(\mathrm{H})=12.0$ and in the notations $[\mathrm{X} / \mathrm{H}]$ and $[\mathrm{X} / \mathrm{Fe}]$.

\begin{tabular}{lcccc}
\hline \hline Species & $n$ & $\log \varepsilon$ & {$[\mathrm{X} / \mathrm{H}]$} & {$[\mathrm{X} / \mathrm{Fe}]$} \\
\hline $\mathrm{Fe}_{\text {I }}$ & 47 & $5.59 \pm 0.09$ & -1.93 & - \\
$\mathrm{Fe}_{\text {II }}$ & 9 & $5.58 \pm 0.08$ & -1.94 & - \\
$\mathrm{C}_{\left(\mathrm{C}_{2}\right)}$ & $\mathrm{syn}$ & $7.21 \pm 0.05$ & -1.31 & +0.62 \\
$\mathrm{~N}(\mathrm{CN})$ & $\mathrm{syn}$ & $6.59 \pm 0.20$ & -1.33 & +0.60 \\
$\mathrm{O}_{\text {I }}$ & 1 & $7.10 \pm 0.03$ & -1.73 & +0.20 \\
$\mathrm{Mg}_{\text {I }}$ & 2 & 6.08 & -1.50 & +0.43 \\
$\mathrm{Si}_{\mathrm{I}}$ & 2 & 6.01 & -1.54 & +0.39 \\
$\mathrm{Ca}_{\text {I }}$ & 11 & $4.72 \pm 0.10$ & -1.64 & +0.29 \\
$\mathrm{Sc}_{\text {II }}$ & 6 & $1.31 \pm 0.27$ & -1.86 & +0.07 \\
$\mathrm{Ti}_{\text {I }}$ & 6 & $3.07 \pm 0.15$ & -1.95 & -0.02 \\
$\mathrm{Cr}_{\text {I }}$ & 5 & $3.63 \pm 0.19$ & -2.04 & -0.11 \\
$\mathrm{Mn}_{\text {I }}$ & 2 & 2.99 & -2.40 & -0.47 \\
$\mathrm{Ni}_{\text {I }}$ & 5 & $4.26 \pm 0.18$ & -1.99 & -0.06 \\
$\mathrm{Zn}_{\text {I }}$ & 2 & 2.79 & -1.81 & +0.12 \\
$\mathrm{Y}_{\text {II }}$ & 4 & $0.24 \pm 0.12$ & -2.00 & -0.07 \\
$\mathrm{Ba}_{\text {II }}$ & 2 & 1.15 & -0.98 & +0.95 \\
$\mathrm{La}_{\text {II }}$ & 3 & $0.00 \pm 0.13$ & -1.17 & +0.76 \\
$\mathrm{Ce}_{\text {II }}$ & 7 & $0.54 \pm 0.12$ & -1.04 & +0.89 \\
$\mathrm{Nd}_{\text {II }}$ & 14 & $0.50 \pm 0.13$ & -1.00 & +0.92 \\
$\mathrm{~Pb}_{\text {I }}$ & 1 & 1.61 & -0.34 & +1.58 \\
\hline
\end{tabular}

Notes. $\mathrm{C} / \mathrm{O}=1.26 \pm 0.15 .{ }^{12} \mathrm{C} /{ }^{13} \mathrm{C}=8.0 \pm 2.0$. The adopted solar abundances are from Grevesse \& Sauval (1998).

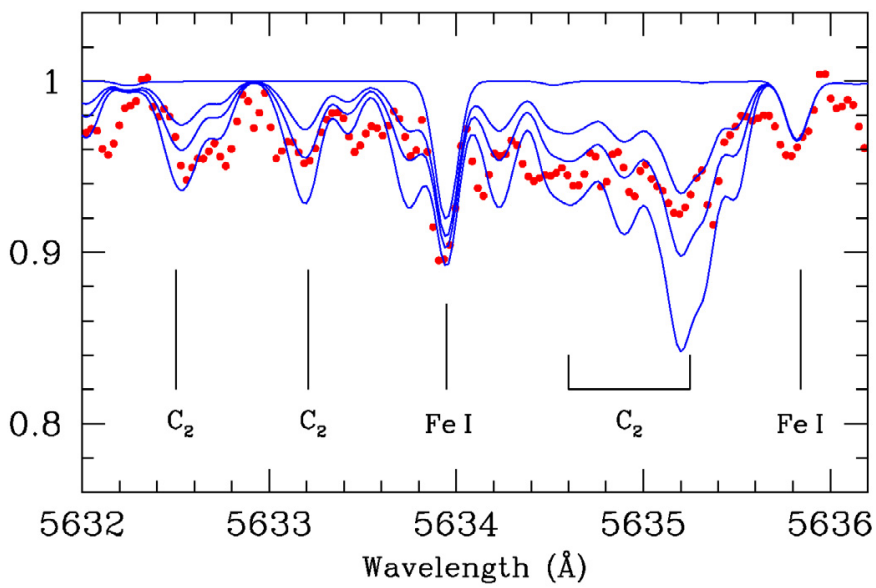

Fig. 2. Observed (dotted red line) and synthetic (solid blue line) spectra in the region around the $C_{2}$ molecule lines at $5635 \AA$. We show the synthesis for the carbon abundances $\log \varepsilon(\mathrm{C})=7.16,7.21$, and 7.26. The upper line corresponds to the spectrum calculated without any contribution from the $\mathrm{C}_{2}$ lines.

Figure 2 shows the observed and synthetic spectra in the region around the $\mathrm{C}_{2}(0,1)$ band head of the Swan system $A^{3} \Pi_{g}-X^{3} \Pi_{u}$ at $\lambda 5635 \AA$. The oxygen abundance was inferred from the [OI] forbidden line at $\lambda 6300.304 \AA$. In our calculations for this line we used the oscillator strength $\log g f=-9.717$ obtained by Allende Prieto et al. (2001) in their analysis of the solar oxygen abundance.

Lithium abundance was derived from the synthetic spectra matches to the Li I $\lambda 6708 \AA$ resonance doublet. Special attention was given to the identification of s-process element lines in the vicinity of the lithium line. Using the databases VALD (Kupka et al. 1999, and references therein) and D.R.E.A.M. (Database on Rare-Earths At Mons University, Biémont et al. 1999) we created a line list that is as complete as possible. For the lines appearing in both databases, the VALD oscillator
Table 5. Abundance uncertainties of the Feh-Duf star.

\begin{tabular}{lccccccc}
\hline \hline Species & $\Delta T_{\text {eff }}$ & $\Delta \log g$ & $\Delta \xi$ & $\Delta[\mathrm{Fe} / \mathrm{H}]$ & $\Delta W_{\lambda}$ & $\left(\sum \sigma^{2}\right)^{1 / 2}$ & $\sigma_{\text {obs }}$ \\
& $+120 \mathrm{~K}$ & +0.2 & +0.3 & +0.1 & $+3 \mathrm{~m} \AA$ & & \\
\hline $\mathrm{Fe}_{\text {I }}$ & +0.20 & -0.03 & -0.10 & +0.04 & +0.06 & 0.23 & 0.09 \\
$\mathrm{Fe}_{\text {II }}$ & -0.03 & +0.07 & -0.04 & -0.01 & +0.06 & 0.10 & 0.08 \\
$\mathrm{Mg}_{\text {I }}$ & +0.23 & -0.06 & -0.17 & +0.04 & +0.05 & 0.30 & - \\
$\mathrm{Si}_{\text {I }}$ & +0.12 & -0.03 & -0.10 & +0.03 & +0.07 & 0.18 & - \\
$\mathrm{Ca}_{\text {I }}$ & +0.17 & -0.03 & -0.08 & +0.03 & +0.05 & 0.20 & 0.10 \\
$\mathrm{Sc}_{\text {II }}$ & +0.04 & +0.07 & -0.06 & -0.02 & +0.05 & 0.11 & 0.27 \\
$\mathrm{Ti}_{\text {I }}$ & +0.24 & -0.03 & -0.09 & +0.04 & +0.05 & 0.27 & 0.15 \\
$\mathrm{Cr}_{\text {I }}$ & +0.25 & -0.03 & -0.08 & +0.03 & +0.05 & 0.27 & 0.19 \\
$\mathrm{Mn}_{\text {I }}$ & +0.18 & -0.03 & -0.06 & +0.02 & +0.03 & 0.20 & - \\
$\mathrm{Ni}_{\text {I }}$ & +0.18 & -0.02 & -0.04 & +0.02 & +0.06 & 0.20 & 0.09 \\
$\mathrm{Zn}_{\text {I }}$ & +0.02 & +0.02 & -0.06 & 0.00 & +0.06 & 0.09 & - \\
$\mathrm{Y}_{\text {II }}$ & +0.06 & +0.05 & -0.10 & -0.02 & +0.05 & 0.14 & 0.12 \\
$\mathrm{Ba}_{\text {II }}$ & +0.12 & +0.05 & -0.27 & -0.02 & +0.04 & 0.30 & - \\
$\mathrm{La} \mathrm{II}$ & +0.08 & +0.07 & -0.03 & -0.04 & +0.06 & 0.13 & 0.13 \\
$\mathrm{Ce}_{\text {II }}$ & +0.07 & +0.05 & -0.12 & -0.01 & +0.05 & 0.16 & 0.12 \\
$\mathrm{Nd}_{\text {II }}$ & +0.07 & +0.06 & -0.05 & -0.02 & +0.03 & 0.11 & 0.13 \\
\hline
\end{tabular}

Notes. The second column gives the variation in the abundance caused by the variation in $T_{\text {eff }}$. The other columns refer, respectively, to the variations due to $\log g, \xi,[\mathrm{Fe} / \mathrm{H}]$ and $W_{\lambda}$. The seventh column gives the compounded rms uncertainty of the second to sixth columns. The last column gives the observed abundance dispersion for those elements whose abundances were derived using more than three lines.

strength values for the s-process element lines were replaced by those from the D.R.E.A.M. database. The CN lines in the vicinity of the Li I doublet were included in the line list. The wavelengths and oscillator strengths for the individual hyperfine and isotopic components of the lithium lines were taken from Smith et al. (1998) and Hobbs et al. (1999). A solar ${ }^{6} \mathrm{Li} /{ }^{7} \mathrm{Li}$ isotopic ratio $\left({ }^{6} \mathrm{Li} /{ }^{7} \mathrm{Li}=0.081\right)$ was adopted in calculations of the synthetic spectrum.

\subsection{Abundance uncertainties}

Table 5 shows that neutral elements are rather sensitive to temperature variations. The singly ionized elements are also sensitive to variations in $\log g$. In the case of elements, such as barium, whose abundance is based on stronger lines, the error introduced by the microturbulence is significant. For the elements analyzed via spectrum synthesis, the same technique was used, by varying $T_{\text {eff }}, \log g, \xi$, and $[\mathrm{Fe} / \mathrm{H}]$ and computing the abundance changes introduced by the variation in these atmospheric parameters. The resulting uncertainties are also included in Table 5.

The abundance uncertainties arising from errors in the equivalent widths measurements were computed using expressions provided by Cayrel (1988). The errors in the equivalent widths are set, essentially, by the $S / N$ and the spectral resolution. In our case, with $R \approx 48000$ and a typical $S / N$ of 150 , the expected uncertainties in the equivalent widths are about 2-3 mA. These error estimates were applied to the measured $E W_{\lambda}$ 's and the corresponding changes in the element abundances are listed in Col. 6 of Table 5 .

We also estimated the influence of model errors, such as the uncertainties in the effective temperatures and surface gravities, on the derived CNO abundances. Moreover, as mentioned above, the abundances of the $\mathrm{CNO}$ elements are interdependent, the uncertainties in the oxygen abundance determination affecting the carbon abundance and vice versa. Uncertainties in the carbon 
Table 6. Effect of errors in atmospheric parameters and carbon and oxygen abundances on the $\mathrm{CNO}$ abundances.

\begin{tabular}{|c|c|c|c|c|c|c|}
\hline Species & $\begin{array}{c}\Delta T_{\text {eff }} \\
+120 \mathrm{~K}\end{array}$ & $\begin{array}{c}\Delta \log g \\
+0.2\end{array}$ & $\begin{array}{c}\Delta \xi \\
+0.3\end{array}$ & $\begin{array}{c}\Delta \log (\mathrm{C}) \\
+0.1\end{array}$ & $\begin{array}{c}\Delta \log (\mathrm{O}) \\
+0.1\end{array}$ & $\sigma_{\text {tot }}$ \\
\hline $\mathrm{C}$ & +0.20 & +0.01 & 0.00 & - & +0.03 & 0.20 \\
\hline $\mathrm{N}$ & +0.33 & 0.00 & 0.00 & -0.12 & +0.05 & 0.35 \\
\hline $\mathrm{O}$ & 0.00 & +0.03 & +0.01 & 0.02 & - & 0.04 \\
\hline
\end{tabular}

Notes. The second to fourth columns give the variation in the abundances due to variations in $T_{\text {eff }}, \log g$, and $\xi$. The fifth and sixth columns shows variations caused by variations in the $\mathrm{C}$ and/or $\mathrm{O}$ abundances.

abundance cause variations in the nitrogen abundance, because the $\mathrm{CN}$ molecule lines were used for the nitrogen abundance determination. The variations in the abundance caused by changes in the effective temperature $( \pm 120 \mathrm{~K})$, surface gravity $( \pm 0.2 \mathrm{dex})$, and both $\mathrm{C}$ and $\mathrm{O}$ abundances $( \pm 0.1 \mathrm{dex})$ are summarized in Table 6. In the last column of this table, we present the resulting abundance uncertainties, $\sigma_{\text {tot }}$, calculated as the square root of the sum of squares of the various sources of uncertainties. Derived CNO abundances are weakly sensitive to variations in the microturbulent velocity because weak lines were used for their determination. A further source of uncertainty in the molecular line analysis is the uncertainty in the molecule constants, in particular the dissociation energy of the $\mathrm{CN}$ molecule. Our tests showed that variations in the $\mathrm{CN}$ molecule dissociation energy of $\Delta D_{0}(\mathrm{CN})=+0.10 \mathrm{eV}$ resulted in variations in the nitrogen abundance of about -0.1 dex. Calculations of the carbon isotopic ratios do not depend on the uncertainties in the $\mathrm{C}$ and $\mathrm{N}$ abundances and molecular parameters. The error in the ${ }^{12} \mathrm{C} /{ }^{13} \mathrm{C}$ determination comes mainly from uncertainties in the observed spectrum, such as possible contamination by unidentified atomic or molecular lines, or uncertainties in the continuum placement.

\section{Discussion}

\subsection{Luminosity and distance of the Feh-Duf star}

Since the parallax of the Feh-Duf star had not been measured, we estimated its luminosity and distance using theoretical evolutionary tracks. Assuming a stellar mass of $0.8 M_{\odot}$ and using the evolution tracks of Fagotto et al. (1994), we obtained $\log L / L_{\odot}=$ $3.01 \pm 0.20$ or $M_{\text {bol }}=-2.8 \pm 0.5$. Taking into consideration the observed magnitude of $V=11.78$ and $B C=-0.495$ (Allonso et al. 1999) we obtained $M_{V}=-2.3 \pm 0.5$ and $r=5.9_{-1.2}^{+1.5} \mathrm{kpc}$. The reddening $E_{B-V}=0.06$ determined for the LMC stars (Grieve \& Madore 1986; Pompéia et al. 2008) was adopted. This value of the colour excess agrees well with the intensity of the interstellar $\mathrm{Na}_{\mathrm{I}} \mathrm{D}_{1}$ and $\mathrm{D}_{2}$ lines of $126.9 \mathrm{~m} \AA$ and $176.1 \mathrm{~m} \AA$, respectively, in the spectrum of the Feh-Duf star. Figure 3 displays the evolutionary tracks for several stellar masses in the $\log g-\log T_{\text {eff }}$ plane at a metallicity of $Z=0.0004$ computed by Fagotto et al. (1994). The position of the Feh-Duf star in this plane is also shown.

According to Table IV of Hartwick \& Cowley (1985), $\mathrm{CH}$ stars have $M_{V}$ values between -0.25 and -2.2 , therefore the Feh-Duf star, with $M_{V}=-2.3$, could be a $\mathrm{CH}$ star and hence a binary star. It indeed displays characteristics of halo population: low metallicity, high radial velocity, and high galactic latitude. However, from the luminosity derived above, we cannot totally rule out the possibility that the Feh-Duf star could be an

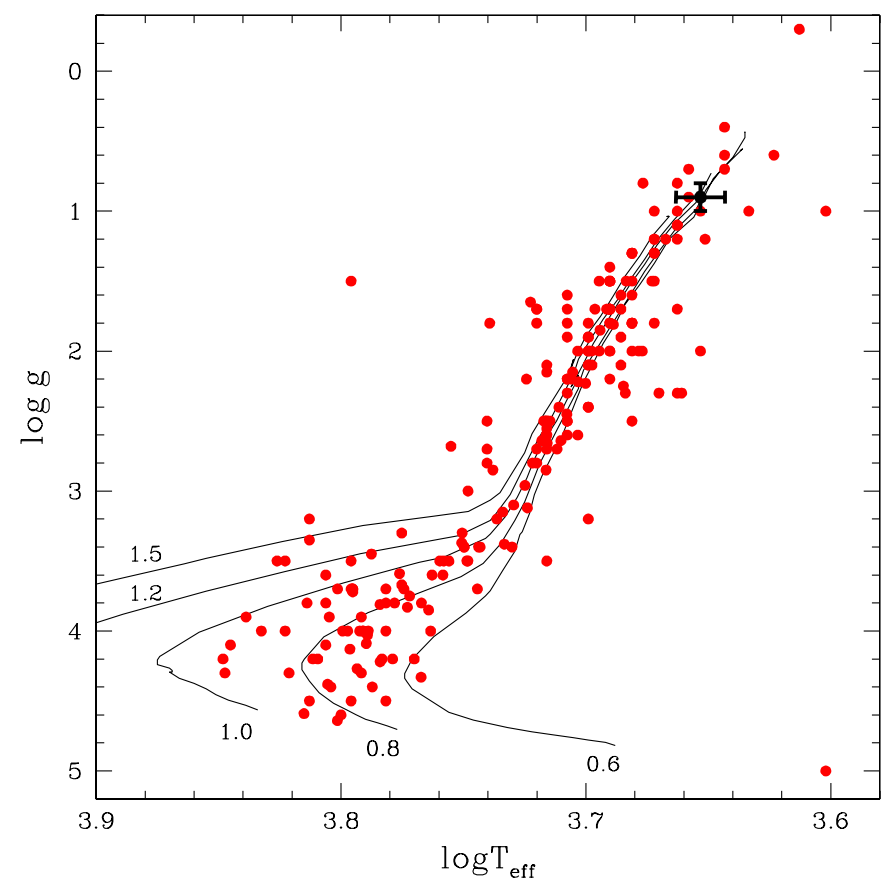

Fig. 3. Location of the Feh-Duf star in the $\left(\log T_{\text {eff }}, \log g\right)$ plane (filled circle with attached error bars). Evolutionary tracks (Fagotto et al. 1994) at metallicity $Z=0.0004$ for stellar masses $0.6,0.8,1.0,1.2$, and $1.5 M_{\odot}$ are plotted. Red circles represent the position of CEMP, $\mathrm{CH}$ and barium stars (Masseron et al. 2010).

early-AGB star. Theoretical calculations show that for a star to be at this phase, it should have a luminosity of $\log L / L_{\odot}=3.1$ $\left(M_{\mathrm{bol}}=-3.1\right.$, Vassiliadis \& Wood 1993).

We determined the radial velocity of the Feh-Duf star to be $v_{\text {rad }}=+448.3 \pm 0.6 \mathrm{~km} \mathrm{~s}^{-1}$. This value is in good agreement with the value $v_{\text {rad }}=+440 \mathrm{~km} \mathrm{~s}^{-1}$ obtained by Fehrenbach \& Duflot (1981).

\subsection{Kinematics}

Assuming a distance of $r=5.9 \mathrm{kpc}$, we calculated the spacevelocity components $\left(U_{0}, V_{0}, W_{0}\right)$ for the Feh-Duf star using the algorithm of Johnson \& Soderblom (1987). Proper motions were taken from NOMAD catalog (Zacharias et al. 2004). The obtained heliocentric space velocities are $\left(U_{0}, V_{0}, W_{0}\right)=$ $(-116,-491,-106) \mathrm{km} \mathrm{s}^{-1}$. We transformed the $V_{0}$ component of the space velocity of the Feh-Duf star to the Galactocentric reference frame $(\mathrm{GRF})$ using $(U, V, W)_{\odot}=(9,232,7)$ (Venn et al. 2004), which corresponds to $V_{\mathrm{GRF}}=-259 \mathrm{~km} \mathrm{~s}^{-1}$. In Table 7, we present the space velocity components of the Feh-Duf star calculated for different (from 0.6 to $1.5 M_{\odot}$ ) values of the stellar mass. The luminosities, and hence the distances, corresponding to the adopted masses were estimated from the above mentioned evolutionary tracks. The values of $V_{\mathrm{GRF}}$ shows that the Feh-Duf star has extreme retrograde motion. As pointed out by Marsakov \& Borkova (2006), a star born in a monotonically collapsing single protogalactic cloud could not be in a retrograde orbit. This extreme retrograde motion of the FehDuf star may be a sign that this star was accreted by the Milky Way from a dwarf satellite galaxy. 
N. A. Drake and C. B. Pereira: The Fehrenbach \& Duflot star

Table 7. Space velocity components of the Feh-Duf star relative to the Sun $\left(U_{0}, V_{0}\right.$, and $\left.W_{0}\right)$ and in the Galactic reference frame ( $\left.V_{\mathrm{GRF}}\right)$ calculated for different distances.

\begin{tabular}{cccccccc}
\hline \hline$M / M_{\odot}$ & $M_{V}$ & $\log L / L_{\odot}$ & $\begin{array}{c}\text { Dist } \\
\mathrm{kpc}\end{array}$ & $\begin{array}{c}U_{0} \\
\mathrm{~km} \mathrm{~s}^{-1}\end{array}$ & $\begin{array}{c}V_{0} \\
\mathrm{~km} \mathrm{~s}^{-1}\end{array}$ & $\begin{array}{c}W_{0} \\
\mathrm{~km} \mathrm{~s}^{-1}\end{array}$ & $\begin{array}{c}V_{\mathrm{GRF}} \\
\mathrm{km} \mathrm{s}^{-1}\end{array}$ \\
\hline 0.6 & -2.0 & 2.88 & 5.1 & -95.0 & -473.2 & -127.0 & -241.2 \\
0.8 & -2.3 & 3.01 & 5.9 & -116.3 & -490.8 & -105.8 & -258.8 \\
1.0 & -2.5 & 3.10 & 6.6 & -134.6 & -505.9 & -87.6 & -273.9 \\
1.5 & -3.0 & 3.28 & 8.1 & -172.6 & -537.4 & -49.7 & -305.4 \\
\hline
\end{tabular}

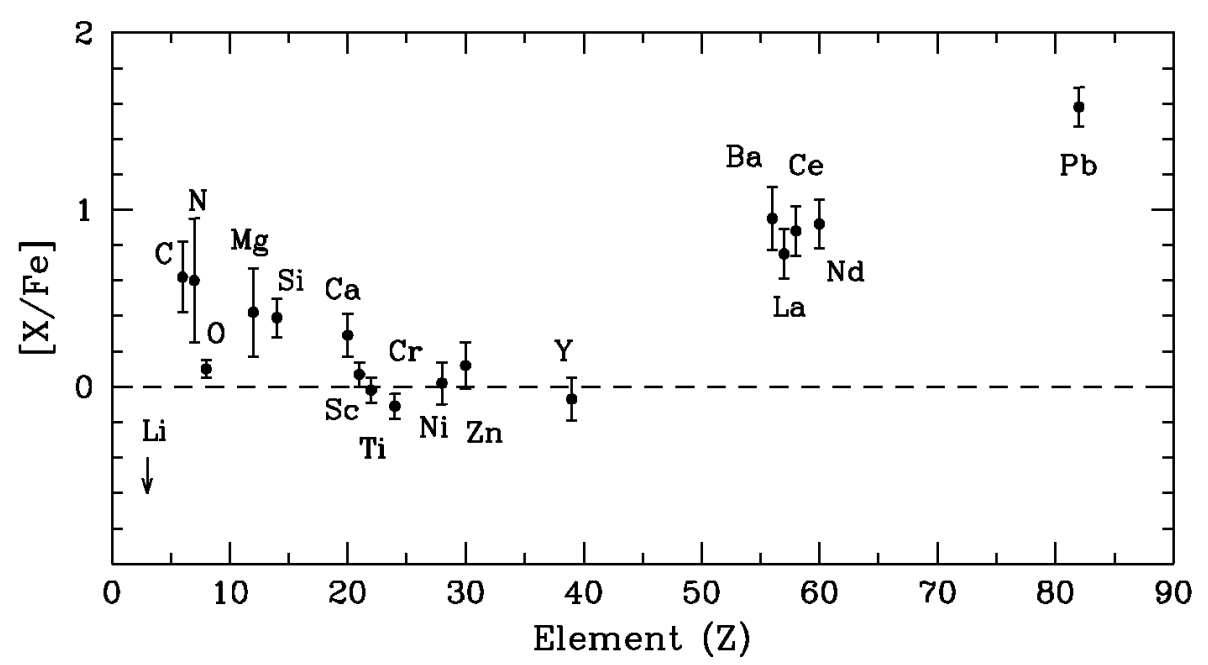

Fig. 4. Abundance pattern of the Feh-Duf star. Errors bars represent the uncertainty estimates described in the text.

\subsection{Abundances}

Below we discuss the abundance pattern of the Feh-Duf star by comparing it with previous studies for some halo population stars and also with the abundance patterns of chemically peculiar stars with heavy-element overabundances already reported in the literature. In addition, we also compare the abundance pattern of the Feh-Duf star with stars exhibiting retrograde motion already analyzed in the literature, as well as with stars belonging to dSph galaxies. Figure 4 shows the abundance pattern of the Feh-Duf star analyzed in this work.

\subsubsection{Nitrogen and oxygen}

Abundance surveys of dwarf stars show that there is no trend for the $[\mathrm{N} / \mathrm{Fe}]$ versus $[\mathrm{Fe} / \mathrm{H}]$ ratio, that is, in the metallicity range $-2.0<[\mathrm{Fe} / \mathrm{H}]<+0.3,[\mathrm{~N} / \mathrm{Fe}]$ is $\approx 0.0$ (Clegg et al. 1981 ; Tomkin \& Lambert 1984; Carbon et al. 1987). As a star becomes a giant, nuclear processed material, due to the deepening of its convective envelope, is brought from the interior to the outer layers of the star changing the surface composition. As a consequence of the first dredge-up process, the abundance of ${ }^{12} \mathrm{C}$ is reduced and the abundance of nitrogen is enhanced (Lambert 1981). The nitrogen-to-iron ratio in the Feh-Duf star is high, $[\mathrm{N} / \mathrm{Fe}]=0.60 \pm 0.20$, although this value is less than the mean $[\mathrm{N} / \mathrm{Fe}]$ ratio observed in the $\mathrm{CH}$ stars analyzed by Vanture (1992b), $[\mathrm{N} / \mathrm{Fe}]=1.3 \pm 0.6$.

Comparison of the oxygen-to-iron ratio in the Feh-Duf star with Galactic halo stars indicates that the $[\mathrm{O} / \mathrm{Fe}]$ ratio in the Feh-Duf star is about 0.3 dex below the corresponding value for the stars of the same metallicity in the Galaxy (Nissen et al. 2002) (see Fig. 5).

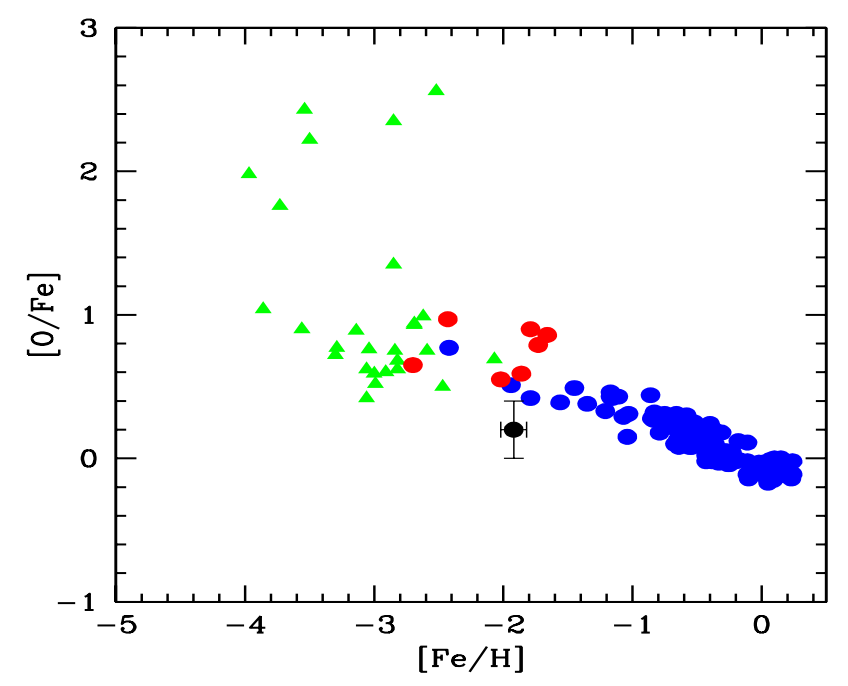

Fig. 5. $[\mathrm{O} / \mathrm{Fe}]$ versus $[\mathrm{Fe} / \mathrm{H}]$ for the Feh-Duf star (black circle), disk stars (blue circles), CEMP-s stars (red circles), and CEMP-no stars (green triangles). Abundance data for disk stars are from Edvardsson et al. (1993) and Nissen et al. (2002). Abundance data for CEMPno stars are from Cayrel et al. (2004) and from Masseron et al. (2010) and abundance data for CEMP-s stars are from Masseron et al. (2010).

\subsection{2. ${ }^{12} \mathrm{C} /{ }^{13} \mathrm{C}$ ratio}

In Fig. 6, we show ${ }^{12} \mathrm{C} /{ }^{16} \mathrm{O}$ versus ${ }^{12} \mathrm{C} /{ }^{13} \mathrm{C}$. In this figure, barium stars (red open squares) occupy a region between the GK-giants and C stars. They closely follow the sequence of GK giants passing through the $\mathrm{S}$ and $\mathrm{SC}$ types to become a carbon star along the TP-AGB phase. In $\mathrm{CH}$ subgiants, the ${ }^{12} \mathrm{C} /{ }^{13} \mathrm{C}$ ratio was investigated by Sneden (1983) and Drake \& Pereira (2007). These two studies show that the ${ }^{12} \mathrm{C} /{ }^{13} \mathrm{C}$ ratio lies between 20 and 50. Compared to giants, this is taken as evidence that the first dredge-up has not yet started in these stars. 


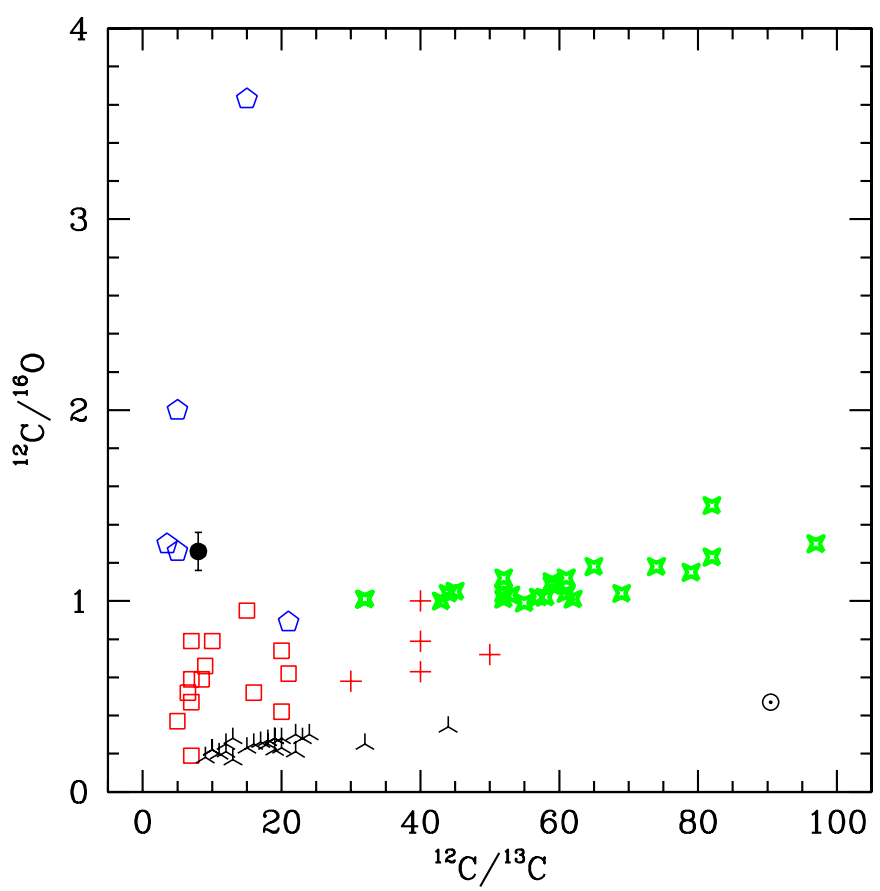

Fig. 6. ${ }^{12} \mathrm{C} /{ }^{16} \mathrm{O}$ versus ${ }^{12} \mathrm{C} /{ }^{13} \mathrm{C}$ ratios for several classes of stars and for the Feh-Duf star. Barium giants (red open squares); disk carbon stars, (green starry points); GK giants, (upside-down "Y"); CH stars, (blue open polygons); subgiant $\mathrm{CH}$ stars, (red plus sign) and Feh-Duf star (filled black circle).

The ${ }^{12} \mathrm{C} /{ }^{13} \mathrm{C}$ ratio in $\mathrm{CH}$ stars was investigated by Vanture (1992a), who showed that these stars could be divided into two groups according to the value of the ${ }^{12} \mathrm{C} /{ }^{13} \mathrm{C}$ ratio, some having ${ }^{12} \mathrm{C} /{ }^{13} \mathrm{C} \approx 3.0$ and others ${ }^{12} \mathrm{C} /{ }^{13} \mathrm{C} \geq 25$.0. For barium giants, the ${ }^{12} \mathrm{C} /{ }^{13} \mathrm{C}$ ratio lies between 8 and 20 (Barbuy et al. 1992; Drake \& Pereira 2008; Pereira \& Drake 2009). It remains to be verified, when the masses of $\mathrm{CH}$ stars and barium stars become available, whether this division in the ${ }^{12} \mathrm{C} /{ }^{13} \mathrm{C}$ ratios is related to mass. The star analyzed in this work, with ${ }^{12} \mathrm{C} /{ }^{13} \mathrm{C}=8.0$, adds only one data point in this diagram but provides evidence that the Feh-Duf star is a $\mathrm{CH}$ star.

\subsubsection{Iron group}

Nickel abundance is expected to follow iron and does indeed with $[\mathrm{Ni} / \mathrm{Fe}]=-0.02$. The $[\mathrm{Ni} / \mathrm{Fe}]$ ratio remains close to 0.0 in a metallicity range from -2.0 to 0.0 (Jonsell et al. 2005). The $[\mathrm{Sc} / \mathrm{Fe}]$ ratio also does not exhibit any trend in the metallicity range $-3.0 \leq[\mathrm{Fe} / \mathrm{H}] \leq 0.0$ (Carretta et al. 2002) and the Feh-Duf star confirms this conclusion. The chromium-toiron ratio also displays no trend for metal-poor stars. For the Feh-Duf star, the $[\mathrm{Cr} / \mathrm{Fe}]$ ratio for the Feh-Duf star has a value typical for a metal-poor star with metallicity $[\mathrm{Fe} / \mathrm{H}] \approx-2.0$. The Mn deficiency is another indication that the Feh-Duf star belongs to a metal-poor population (Gratton 1989; Carretta et al. 2002). The Mn deficiency observed in the atmosphere of the Feh-Duf star is typical of halo field and globular clusters stars (Sobeck et al. 2006). Zinc was analyzed by Sneden et al. (1991) for disk and halo stars. Zinc abundance also follows the trend seen in the metallicity range between -2.5 and -0.5 .

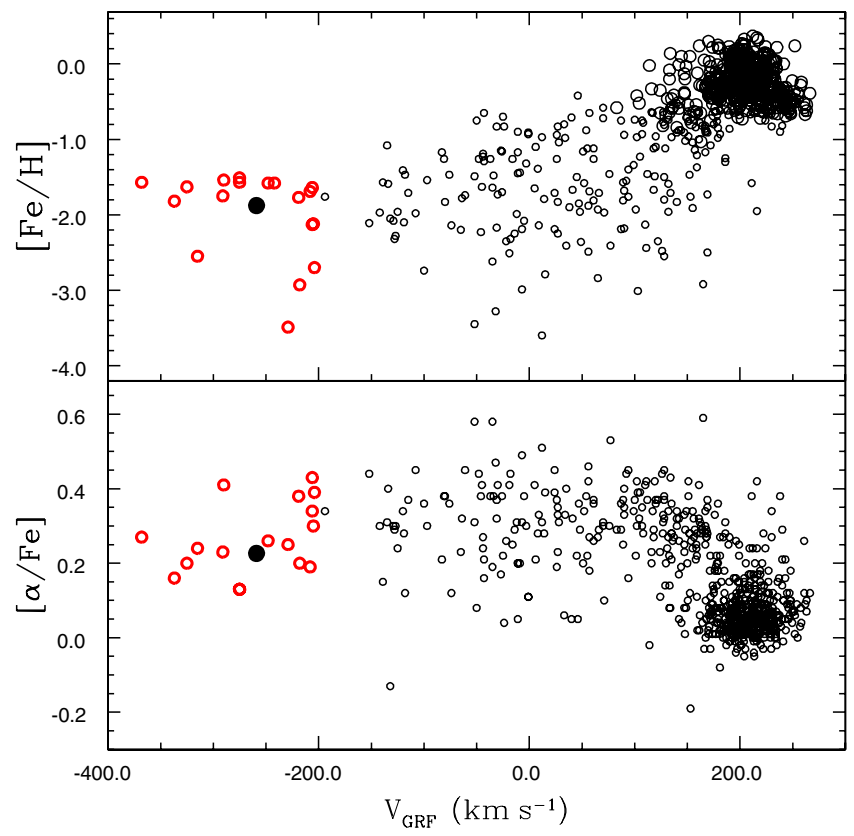

Fig. 7. Metallicity (top) and $[\alpha / \mathrm{Fe}]$ (bottom) versus the $V$ component of the space velocity relative to the Galactocentric Reference Frame for stars in the thin and thick discs, in the halo (black open circles) and those that exhibit extreme retrograde motion (red open circles). Filled black circle represents the Feh-Duf star.

\subsection{4. $\alpha$-elements}

The Feh-Duf star presents $\alpha$-element abundance $([\mathrm{Mg}+\mathrm{Ca}+\mathrm{Ti} /$ $3 \mathrm{Fe}]=0.23$ ) lower than halo stars of the same metallicity (Carretta et al. 2002). Venn et al. (2004) analysed the chemical abundances of the stars in both the Galaxy and dwarf spheroidal (dSph) satellite galaxies. They confirmed that the $[\alpha / \mathrm{Fe}]$ ratios in most stars of dSph galaxies are lower than in Galactic stars of similar metallicity. Figure 2 of Venn et al. (2004) shows that dSph stars are clearly separated from the majority of Galactic disk and halo stars. They also found that dSph's stars overlap with those stars of the Galactic halo that are in extreme retrograde orbits $\left(V_{\mathrm{GRF}}<-200 \mathrm{~km} \mathrm{~s}^{-1}\right)$. In Fig. 7, we reproduce Fig. 1 of Venn et al. (2004), where we see the position of the Feh-Duf star in the planes metallicity $([\mathrm{Fe} / \mathrm{H}])$ versus $V_{\mathrm{GRF}}$ and $[\alpha / \mathrm{Fe}]$ versus $V_{\mathrm{GRF}}$. These diagrams and the low oxygen abundance seen in the Feh-Duf star support the hypothesis that this star may have been accreted by the Milky Way from a dwarf satellite galaxy.

\subsubsection{Carbon and s-process elements}

Figure 8 shows the $[\mathrm{C} / \mathrm{Fe}]$ abundance ratio plotted as a function of the metallicity $[\mathrm{Fe} / \mathrm{H}]$ for barium stars (giants and dwarfs), for $\mathrm{CH}$ stars, and for CEMP stars. We note that the Feh-Duf star has a lower $[\mathrm{C} / \mathrm{Fe}]$ ratio as "expected" for a chemically peculiar binary star seen in this figure.

Figure 9 shows the $[\mathrm{s} / \mathrm{Fe}]$ abundance ratio plotted as a function of the metallicity given by $[\mathrm{Fe} / \mathrm{H}]$ for the same objects as in Fig. 8, for yellow symbiotic stars, and for the Feh-Duf star. Here "s" represents the mean of the elements created by slow neutron capture reactions (s-process): $\mathrm{Y}, \mathrm{Ba}, \mathrm{La}, \mathrm{Ce}, \mathrm{Nd}$, and $\mathrm{Pb}$. Among CEMP stars, there are six that are binaries i.e. CS $22942-$ 019, CS 22948-027, CS 29497-030, CS 29497-034, CS 22964161, and HE 0024-2523, The data for these stars concerning their heavy-element $(Z>56)$ overabundances and binarity have 
N. A. Drake and C. B. Pereira: The Fehrenbach \& Duflot star

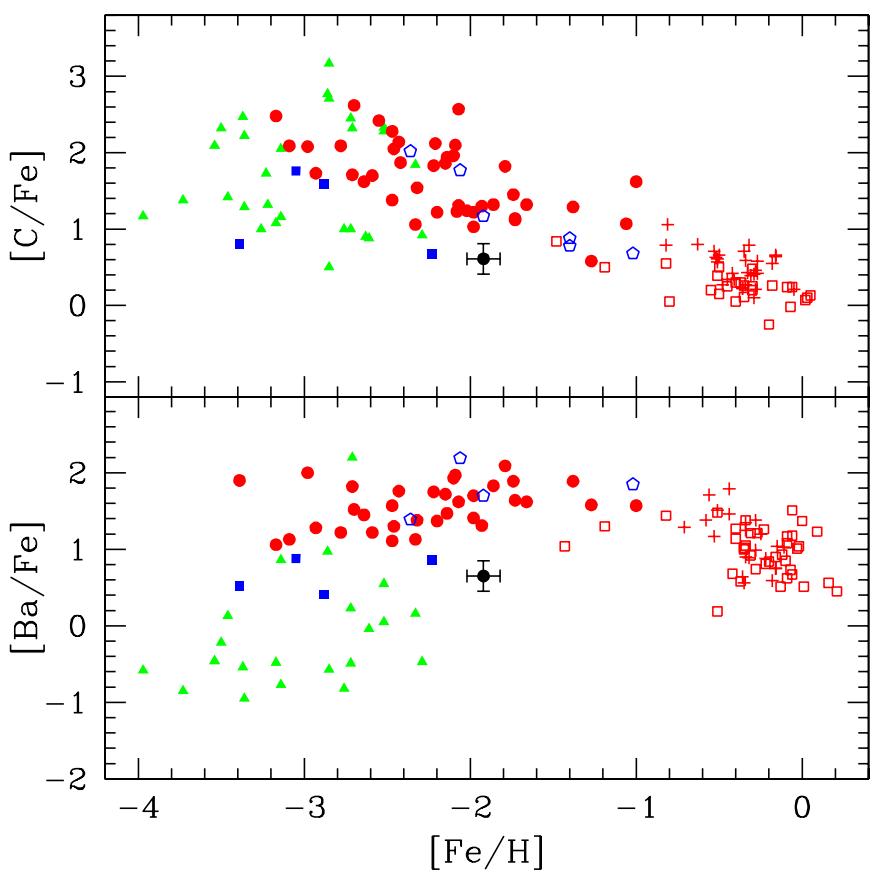

Fig. 8. Diagram of $[\mathrm{C} / \mathrm{Fe}]$ versus $[\mathrm{Fe} / \mathrm{H}]$ for several classes of chemically peculiar binary stars. Barium giants (red open squares); $\mathrm{CH}$ stars (blue open polygons); subgiant $\mathrm{CH}$ stars (red plus sign); and the FehDuf star (filled black circle). We also show the CEMP-s stars (red filled circles), the CEMP-no stars (green triangles) and the CEMP-low-s stars (Masseron et al. 2010) (blue filled squares).

been taken from the recent results by Preston \& Sneden (2001), Sivarani et al. (2004), Barbuy et al. (2005), Lucatello et al. (2003), Thompson et al. (2008), Aoki et al. (2002), and Hill et al. (2000). As we can see from this figure, the Feh-Duf star is enhanced in the s-process elements relative to metal-poor field stars. In the Feh-Duf star, the [element/Fe] ratio for each of these elements, except yttrium, is much higher than the same [element/Fe] ratios measured for metal-poor dwarfs, subgiant, and giant stars analysed by Gratton \& Sneden (1994). The mean values of $[\mathrm{Ba} / \mathrm{Fe}],[\mathrm{La} / \mathrm{Fe}],[\mathrm{Ce} / \mathrm{Fe}]$, and $[\mathrm{Nd} / \mathrm{Fe}]$ at the metallicity of the Feh-Duf star is 0.0 , while the same values for this star are $+0.95,+0.76,+0.89$, and +0.92 , respectively. However, the abundance of light s-process elements, such as Y, is too low for a chemically peculiar binary system, such as $\mathrm{CH}$ stars and barium stars. In Table 2 of Busso et al. (2001), the ratio [1s/Fe] ("light s-elements") given by the mean abundance of $\mathrm{Y}$ and $\mathrm{Zr}$, lies in the range between +0.2 and +1.3 . In the Feh-Duf star, this ratio is given only by the $[\mathrm{Y} / \mathrm{Fe}]$ ratio $([\mathrm{Y} / \mathrm{Fe}]=-0.02)$. According to Clayton (1988), Busso et al. (1999), Travaglio et al. (2004), and Venn et al. (2004), the neutron-capture nucleosynthesis in AGB stars is metallicity dependent. The first-peak elements (such as $\mathrm{Y}$ and $\mathrm{Zr}$ ) are bypassed in favor of the secondpeak elements and those from the third peak. The observed high $[\mathrm{Ba} / \mathrm{Y}]$ ratio is consistent with the expectations for metalpoor AGB s-process yields. For the $[\mathrm{Pb} / \mathrm{Ce}]$ ratio the protonmixing scenario confirms the trend seen in several $\mathrm{CH}$ stars with high lead abundances (Van Eck et al. 2003; Goriely \& Mowlavi $2000)$, although there is still a scatter in the $[\mathrm{Pb} / \mathrm{Ba}]$ ratio for lowmetallicity stars down to $[\mathrm{Fe} / \mathrm{H}]=-2.5$ (Masseron et al. 2010).

The lead abundance was determined using a spectral synthesis technique. Figure 10 shows the observed and synthetic spectra of the Feh-Duf star around $\mathrm{Pb}_{\mathrm{I}} \lambda 4058 \AA$. In Fig. 11, we show the $[\mathrm{Pb} / \mathrm{Ce}]$ ratio as a function of metallicity $([\mathrm{Fe} / \mathrm{H}])$.

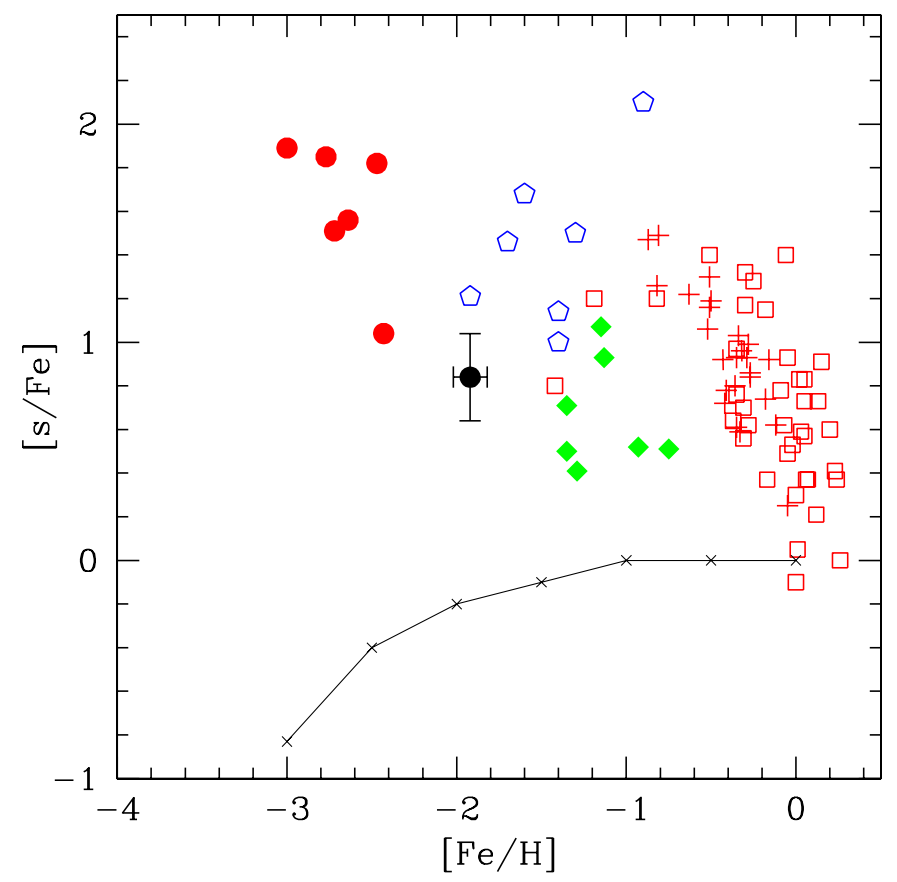

Fig. 9. Diagram of $[\mathrm{s} / \mathrm{Fe}]$ versus $[\mathrm{Fe} / \mathrm{H}]$. The solid line is the mean $\langle[\mathrm{s} / \mathrm{Fe}]\rangle$ for field stars (Gratton \& Sneden 1994; Ryan et al. 1996; and François et al. 2003). Green filled diamonds correspond to yellowsymbiotics, other symbols have the same meaning as in Fig. 8. For the CEMP-s stars, we show only those that have already been proven to be binary systems.

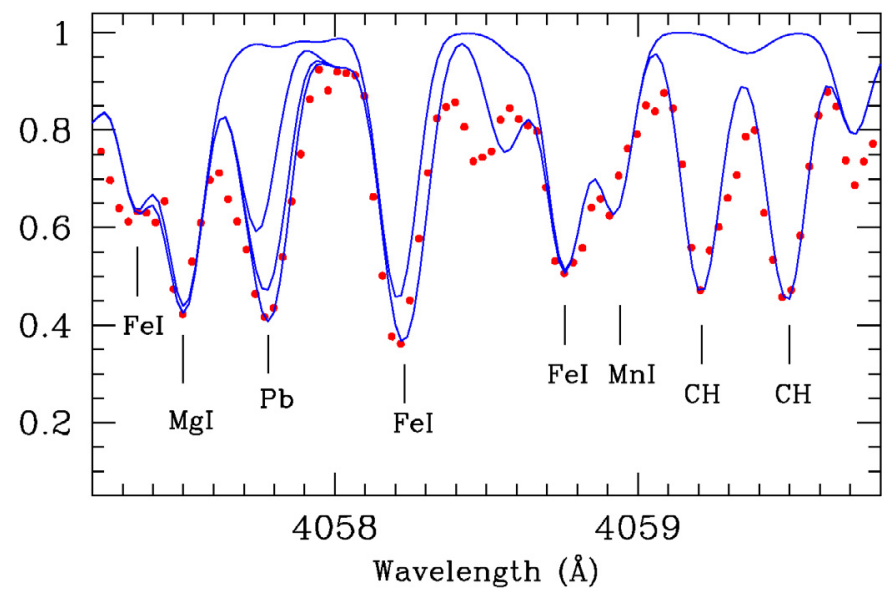

Fig. 10. Observed (dots) and synthetic (lines) spectra of Feh-Duf in the region around the $\mathrm{Pb}$ I line at $\lambda 4057.8 \AA$. The synthetic spectra are shown for lead abundances of $\log \varepsilon(\mathrm{Pb})=0.10,1.40$, and 1.75. The upper line shows a synthesis without contribution from the $\mathrm{CH}$ molecule lines.

In this figure, we show the Feh-Duf star (the filled black circle at $[\mathrm{Fe} / \mathrm{H}]=-1.93$ ), the $\mathrm{CH}$ stars analyzed by Van Eck et al. (2003), CEMP stars that are members of binary systems, and the barium giants and dwarfs from Allen \& Barbuy (2006). The solid line represents the prediction from the standard partial mixing (PM) model as given by Goriely \& Mowlavi (2000). The position of the Feh-Duf in this diagram, follows the same trend as the other barium dwarfs and giants investigated so far. 


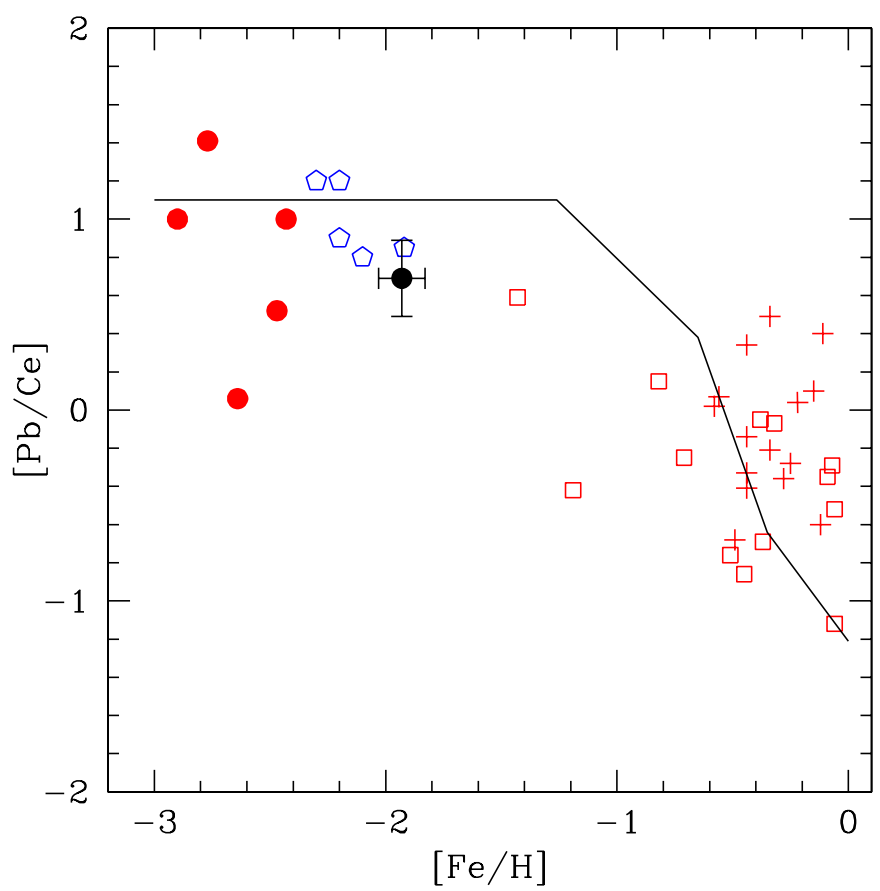

Fig. 11. The behaviour of the $[\mathrm{Pb} / \mathrm{Ce}]$ ratio with metallicity. Symbols have the same meaning as in Fig. 8. The solid line represents the prediction from the standard partial mixing (PM) model as given by Goriely \& Mowlavi (2000). For CEMP-s stars, we considered only those that have already been proven to be binary systems.

\section{The Feh-Duf star compared with $\mathrm{CH}$ and CEMP stars}

As shown above, the Feh-Duf is a low-metallicity star with high carbon and s-element abundances. Therefore, it is interesting to compare it with the $\mathrm{CH}$ and some CEMP stars already investigated in the literature. Unfortunately, we were unable to detect any Eu lines suitable for abundance determination of this element, which is mainly produced by the r-process, and permits us to separate different subclasses of CEMP stars according to the [Ba/Eu] ratio (Beers \& Christlieb 2005; Masseron et al. 2010).

Besides to be an extragalactic candidate due to its observed kinematics and low $[\mathrm{O} / \mathrm{Fe}]$ and $[\alpha / \mathrm{Fe}]$ ratios relative to metalpoor stars in the Galaxy, the abundance pattern of the Feh-Duf star presents another peculiarity, namely, a lower barium and carbon abundance compared to CEMP stars at this metallicity. Figure 8 shows the $[\mathrm{C} / \mathrm{Fe}]$ and $[\mathrm{Ba} / \mathrm{Fe}]$ ratios plotted as a function of metallicity for CEMP stars (CEMP-no, CEMP-s, and CEMP-low-s) from Masseron et al. (2010). In both diagrams of Fig. 8, the Feh-Duf star is slightly below the CEMP stars. From its position in the $\log g-\log T_{\text {eff }}$ plane, we see that the Feh-Duf star is one of the most evolved objects compared to stars in the sample of CEMP stars. In a way similar to CS 30322-023, which is another evolved object (Masseron et al. 2006), its carbon and barium enhancement is only moderate, permiting us to classify Feh-Duf as a CEMP-low-s star. As shown by Shetrone et al. (2010), the $[\mathrm{C} / \mathrm{Fe}]$ ratio shows a plateau at low luminosity, followed by a decline in stars brighter than the "bump" of the RGB luminosity function caused by extra mixing acting after the bump. This extra mixing (regardless of its physical mechanism) may be responsible for the decrease in carbon and s-element abundances in the photospheres of the most evolved CEMP stars.

\section{Conclusions}

The abundance analysis performed by employing highresolution optical spectra of the Feh-Duf star helped us to obtain its abundance pattern and carbon isotopic ratio. Our main conclusions can be summarized as follows:

1. The extreme retrograde motion $\left(V_{\mathrm{GRF}}=-259 \mathrm{~km} \mathrm{~s}^{-1}\right)$ and chemical abundances of the $\alpha$ - and light-s elements distinct from those of Galactic halo stars of the same metallicity may suggest that the Feh-Duf star has an extragalactic origin and was captured by the Milky Way. It is interesting to notice that the Feh-Duf star displays similar trends for its s-process element abundances as the extragalactic carbon star, ALW-C7, analyzed by Abia et al. (2008): i.e. a lower Y and Zr abundances compared to heavy s-elements.

2. We have assumed that the Feh-Duf star has a mass of $0.8 M_{\odot}$ to be compatible with the low metallicity, $[\mathrm{Fe} / \mathrm{H}]=-1.93$, determined for this star. The Feh-Duf star can either be a $\mathrm{CH}$ star (with $\mathrm{C} / \mathrm{O}=1.26$ ) or a star at the early AGB phase since its luminosity is compatible with a star at this phase. In addition, as shown by Masseron et al. (2006), it is not improbable to find an AGB star in the halo. The luminosity of the Feh-Duf star is at the low limit of luminosities of AGB stars, with luminosities exceeding $\log \left(L / L_{\odot}\right) \sim 3-4$ (Ryan et al. 2005). The Feh-Duf star has no detected radial velocity variation (although this conclusion is based on only two measurements). However, as shown by Lucatello et al. (2005) who analysed the observed radial velocity data for a sample of 19 CEMP- $s$ stars, an observed binary fraction of $68 \%$ is consistent with all CEMP- $s$ stars being in multiple systems.

3. The Feh-Duf star is also a "lead star" since its lead-to-cerium ratio is high $([\mathrm{Pb} / \mathrm{Ce}]=+0.70)$ and closely follows the theoretical predictions for a star of this metallicity.

\section{References}

Abia, C., de Laverny, P., \& Wahlin, R. 2008, A\&A, 481, 161

Allen, D. M., \& Barbuy, B. 2006, A\&A, 454, 895

Allende Prieto, C., Lambert, D. L., \& Asplund, M. 2001, ApJ, 556, L63

Allonso, A., Arribas, S., \& Martínez-Roger, C. 1999, A\&AS, 140, 261

Aoki, W., Ryan, S. G., Norris, J. E., et al. 2002, ApJ, 580, 1149

Aoki, W., Beers, T. C., Christlieb, N., et al. 2007, ApJ, 655, 492

Barbuy, B., Jorissen, A., Rossi, S. C. F., \& Arnould, M. 1992, A\&A, 262, 216

Barbuy, B., Spite, M., Spite, F., et al. 2005, A\&A, 429, 1031

Beers, T. C., \& Christlieb, N. 2005, ARA\&A, 43, 531

Biémont, E., \& Godefroid, M. 1980, A\&A, 84, 361

Biémont, E., Grevesse, N., Hannaford, P., \& Lowe, R. M. 1981, ApJ, 248, 867

Biémont, E., Palmeri, P., \& Quinet, P. 1999, Ap\&SS, 269, 635

Busso, M., Gallino, R., \& Wasserburg, G. J. 1999, ARA\&A, 37, 239

Busso, M., Gallino, R., Lambert, D. L., Travaglio, C., \& Smith, V. V. 2001, ApJ, 557,802

Carbon, D. F., Barbuy, B., Kraft, R. P., et al. 1987, PASP, 99, 335

Carretta, E., Gratton, R., Cohen, J. G., Beers, T. C., \& Christlieb, N. 2002, AJ, 124,481

Cayrel, R. 1988, Data Analysis, in The Impact of Very High S/N Spectroscopy on Stellar Physics (Dordrecht: Kluwer), ed. G. Cayrel de Strobel, \& M. Spite, 345

Cayrel, R., Depagne, E., Spite, M., et al. 2004, A\&A, 416, 1117

Clayton, D. D. 1988, MNRAS, 234, 1

Clegg, R. E. S., Tomkin, J., \& Lambert, D. L. 1981, ApJ, 250, 262

Costes, M., Naulin, C., \& Dorthe, G. 1990, A\&A, 232, 270

del Peloso, E. F., Cunha, K., da Silva, L., \& Porto de Mello, G. F. 2005, A\&A, 441, 1149

Depagne, E., Hill, V., Spite, M., et al. 2002, A\&A, 390, 187

Drake, N. A., \& Pereira, C. B. 2007, CNO and Li abundances in Barium-enriched stars, in Convection in Astrophysics, ed. F. Kupka, I. Roxburgh, \& K. Chan, 304

Drake, N. A., \& Pereira, C. B. 2008, AJ, 135, 1070 
Drake, J. J., \& Smith, G. 1991, MNRAS, 250, 89

Edvardsson, B., Andersen, J., Gustafsson, B., et al. 1993, A\&A, 275, 101 Engleman, R. Jr., \& Rouse, P. E. 1975, J. Quant. Spectrosc. Rad. Transf., 15, 831

Fagotto, F., Bressan, A., Bertelli, G., \& Chiosi, C. 1994, A\&AS, 104, 365

Fehrenbach, C., \& Duflot, M. 1981, A\&A, 101, 226

François, P., Depagne, E., Hill, V., et al. 2003, A\&A, 403, 1105

Goriely, S., \& Mowlavi, N. 2000, A\&A, 362, 599

Gratton, R. G. 1989, A\&A, 208, 171

Gratton, R. G., \& Sneden, C. 1988, A\&A, 204, 193

Gratton, R. G., \& Sneden, C. 1994, A\&A, 287, 927

Grevesse, N., \& Sauval, A. J. 1998, Space Sci. Rev., 85, 161

Grieve, G. R., \& Madore, B. F. 1986, ApJS, 62, 427

Hartwick, F. D. A., \& Cowley, A. P. 1985, AJ, 90, 2244

Hannaford, P., Lowe, R. M., Grevesse, N., Biémont, E., \& Whaling, W. 1982, ApJ, 261, 736

Hill, V., Barbuy, B., Spite, M., et al. 2000, A\&A, 353, 557

Hobbs, L. M., Thorburn, J. A., \& Rebull, L. M. 1999, ApJ, 523, 797

Huang, Y., Barts, S. A., \& Halpern, J. B. 1992, J. Phys. Chem., 96, 425

Johnson, D. R. H., \& Soderblom, D. R. 1987, AJ, 93, 864

Jonsell, K., Edvardsson, B., Gustafsson, B., et al. 2005, A\&A, 440, 321

Kaufer, A., Stahl, O. Tubbesing, S., et al. 1999, The Messenger, 95, 8

Korn, A. J., Shi, J., \& Gehren, T. 2003, A\&A, 407, 691

Kupka, F., Piskunov, N. E., Ryabchikova, T. A., Stempels, H. C., \& Weiss, W. W. 1999, A\&AS, 138, 119

Kurucz, R. L. 1993, CD-ROM 13, Atlas9 Stellar Atmosphere Programs and $2 \mathrm{~km} \mathrm{~s}^{-1}$ Grid, Cambridge, Smithsonian Astrophys. Obs

Lambert, D. L. 1981, The chemical composition of red giants - The first dredge-up phase, ed. I. Iben, \& A. Renzini, in Physical Process in Red Giants (Dordrecht: D. Reidel Publ. Co.), 115

Lambert, D. L., Heath, J. E., Lemke, M., \& Drake, J. 1996, ApJS, 103, 183

Lucatello, S., Gratton, R., Cohen, J. G., et al. 2003, AJ, 125, 875

Lucatello, S., Tsangarides, S., Beers, T. C., et al. 2005, ApJ, 625, 825

McWilliam, A., Preston, G. W., Sneden, C., \& Searle, L. 1995, AJ, 109, 2757

Marsakov, V. A., \& Borkova, T. V. 2006, Astron. Lett., 32, 545

Martin, G. A., Fuhr, J. R., \& Wiese, W. L. 1988, J. Phys. Chem. Ref. Data, 17, 4

Mashonkina, L., Gehren, T., Shi, J.-R., Korn, A. J., \& Grupp, F. 2011, A\&A, 528, A87
Masseron, T., van Eck, S., Famaey, B., et al. 2006, A\&A, 455, 1059

Masseron, T., Johnson, J. A., Blez, B., et al. 2010, A\&A, 509, 28

Nissen, P. E., Primas, F., Asplund, M., \& Lambert, D. L. 2002, A\&A, 390, 235

Pereira, C. B., \& Drake, N. A. 2009, A\&A, 496, 791

Pompéia, L., Hill, V., Spite, M., et al. 2008, A\&A, 480, 379

Pradhan, A. D., Partridge, H., \& Bauschlicher, C. W. 1994, J. Chem. Phys., 101, 3857

Preston, G. W., \& Sneden, C. 2001, ApJ, 122, 1545

Reddy, B. E., Tomkin, J., Lambert, D. L., \& Allende Prieto, C. 2003, MNRAS, 340,304

Reyniers, M., Van Winckel, H., Gallino, R., \& Straniero, O. 2004, A\&A, 417, 269

Ryan, S. G., Norris, J. E., \& Beers, T. C. 1996, ApJ, 471, 254

Ryan, S. G., Aoki, W., Norris, J. E., \& Beers, T. C. 2005, ApJ, 635, 349

Shetrone, M., Martell, S. L., Wilkerson, R., et al. 2010, AJ, 140, 1119

Sivarani, T., Bonifacio, P., Molaro, P., et al. 2004, A\&A, 413, 1073

Smith, G., Edvardsson, B., \& Frisk, U. 1986, A\&A, 165, 126

Smith, V. V., Cunha, K., Jorissen, A., \& Boffin, H. M. J. 1996, A\&A, 315, 179

Smith, V. V., Lambert, D. L., \& Nissen, P. E. 1998, ApJ, 506, 405

Sneden, C. 1973, Ph.D. Thesis, Univ. of Texas

Sneden, C. 1983, PASP, 95, 745

Sneden, C., Gratton, R. G., \& Crocker, D. A. 1991, A\&A, 246, 354

Sneden, C., McWilliam, A., Preston, G. W., et al. 1996, ApJ, 467, 819

Sobeck, J. S., Ivans, I. I., Simmerer, J. A., et al. 2006, AJ, 131, 2949

Thévenin, F., \& Idiart, T. P. 1999, ApJ, 521, 753

Thompson, I. B., Ivans, I. I., Bisterzo, S., et al. 2008, ApJ, 677, 556

Tomkin, J., \& Lambert, D. L. 1984, ApJ, 279, 220

Travaglio, C., Gallino, R., Arnone, E., et al. 2004, ApJ, 601, 864

van Winckel, H., \& Reyniers, M. 2000, A\&A, 354, 135

Van Eck, S., Goriely, S., Jorissen, A., \& Plez, B. 2003, A\&A, 404, 291

Vanture, A. 1992a, AJ, 103, 2035

Vanture, A. 1992b, AJ, 104, 1986

Vassiliadis, E., \& Wood, P. R. 1993, ApJ, 413, 641

Venn, K. A., Irwin, M., Shetrone, M. D., et al. 2004, AJ, 128, 1177

Wiese, W. L., \& Martin, G. A. 1980, NSDRS-NBS, 68

Wiese, W. L., Smith, M. W., \& Miles, B. M. 1969, NBS Ref. Data. Ser.

Zacharias, N., Monet, D. G., Levine, S. E., et al. 2004, BAAS, 36, 1418 
Table 1. Observed Fe I and Fe II lines. The $\log g f$ values were taken from Lambert et al. (1996).

\begin{tabular}{|c|c|c|c|c|c|}
\hline Element & $\lambda(\AA)$ & $\chi(\mathrm{eV})$ & $\log g f$ & $E W_{\lambda}(\mathrm{m} \AA)$ & $\log \varepsilon$ \\
\hline \multirow[t]{47}{*}{$\mathrm{Fe}_{\mathrm{I}}$} & 5150.84 & 0.99 & -3.000 & 138 & 5.72 \\
\hline & 5198.71 & 2.22 & -2.140 & 93 & 5.69 \\
\hline & 5232.94 & 2.94 & -0.080 & 143 & 5.55 \\
\hline & 5242.49 & 3.63 & -0.970 & 55 & 5.62 \\
\hline & 5281.79 & 3.04 & -0.830 & 101 & 5.58 \\
\hline & 5288.52 & 3.69 & -1.510 & 17 & 5.46 \\
\hline & 5302.31 & 3.28 & -0.740 & 97 & 5.73 \\
\hline & 5307.36 & 1.61 & -2.970 & 87 & 5.55 \\
\hline & 5322.04 & 2.28 & -2.840 & 42 & 5.54 \\
\hline & 5339.93 & 3.27 & -0.680 & 95 & 5.60 \\
\hline & 5364.87 & 4.45 & 0.230 & 62 & 5.55 \\
\hline & 5369.96 & 4.37 & 0.540 & 77 & 5.43 \\
\hline & 5373.71 & 4.47 & -0.710 & 21 & 5.72 \\
\hline & 5389.48 & 4.42 & -0.250 & 34 & 5.48 \\
\hline & 5445.04 & 4.39 & 0.041 & 65 & 5.71 \\
\hline & 5506.78 & 0.99 & -2.800 & 144 & 5.48 \\
\hline & 5554.90 & 4.55 & -0.380 & 29 & 5.65 \\
\hline & 5567.39 & 2.61 & -2.560 & 38 & 5.57 \\
\hline & 5569.62 & 3.42 & -0.490 & 95 & 5.55 \\
\hline & 5572.84 & 3.40 & -0.280 & 106 & 5.52 \\
\hline & 5576.09 & 3.43 & -0.850 & 79 & 5.61 \\
\hline & 5638.26 & 4.22 & -0.720 & 24 & 5.47 \\
\hline & 5686.53 & 4.55 & -0.450 & 22 & 5.55 \\
\hline & 5717.83 & 4.28 & -0.979 & 20 & 5.70 \\
\hline & 5762.99 & 4.21 & -0.410 & 46 & 5.57 \\
\hline & 6024.06 & 4.55 & -0.060 & 52 & 5.72 \\
\hline & 6027.05 & 4.08 & -1.090 & 22 & 5.58 \\
\hline & 6056.01 & 4.73 & -0.400 & 20 & 5.65 \\
\hline & 6065.48 & 2.61 & -1.530 & 106 & 5.61 \\
\hline & 6136.61 & 2.45 & -1.400 & 133 & 5.74 \\
\hline & 6137.69 & 2.59 & -1.400 & 114 & 5.59 \\
\hline & 6151.62 & 2.18 & -3.290 & 30 & 5.54 \\
\hline & 6173.34 & 2.22 & -2.880 & 53 & 5.58 \\
\hline & 6191.56 & 2.43 & -1.420 & 118 & 5.45 \\
\hline & 6200.32 & 2.60 & -2.440 & 47 & 5.53 \\
\hline & 6213.43 & 2.22 & -2.480 & 73 & 5.48 \\
\hline & 6230.72 & 2.56 & -1.280 & 122 & 5.56 \\
\hline & 6252.56 & 2.40 & -1.720 & 109 & 5.55 \\
\hline & 6265.13 & 2.18 & -2.550 & 87 & 5.71 \\
\hline & 6322.69 & 2.59 & -2.430 & 51 & 5.56 \\
\hline & 6393.60 & 2.43 & -1.430 & 116 & 5.39 \\
\hline & 6419.95 & 4.73 & -0.090 & 32 & 5.59 \\
\hline & 6421.35 & 2.28 & -2.010 & 106 & 5.60 \\
\hline & 6430.85 & 2.18 & -2.010 & 120 & 5.68 \\
\hline & 6592.91 & 2.72 & -1.470 & 95 & 5.43 \\
\hline & 6593.87 & 2.44 & -2.420 & 78 & 5.73 \\
\hline & 6750.15 & 2.42 & -2.620 & 67 & 5.73 \\
\hline \multirow[t]{9}{*}{$\mathrm{Fe}_{\text {II }}$} & 5197.56 & 3.23 & -2.250 & 75 & 5.60 \\
\hline & 5234.62 & 3.22 & -2.240 & 78 & 5.63 \\
\hline & 5284.10 & 2.89 & -3.010 & 48 & 5.41 \\
\hline & 5325.56 & 3.22 & -3.170 & 32 & 5.66 \\
\hline & 5425.25 & 3.20 & -3.210 & 30 & 5.63 \\
\hline & 5534.83 & 3.25 & -2.770 & 48 & 5.58 \\
\hline & 6149.25 & 3.89 & -2.720 & 18 & 5.62 \\
\hline & 6247.55 & 3.89 & -2.340 & 27 & 5.47 \\
\hline & 6432.68 & 2.89 & -3.580 & 32 & 5.58 \\
\hline
\end{tabular}

Table 3. Other lines studied.

\begin{tabular}{|c|c|c|c|c|c|c|}
\hline$\lambda(\AA)$ & Element & $\chi(\mathrm{eV})$ & $\log g f$ & Ref. & $E W_{\lambda}(\mathrm{m} \AA)$ & $\log \varepsilon$ \\
\hline 4571.10 & $\mathrm{Mg}_{\mathrm{I}}$ & 0.00 & $2.042 \mathrm{e}-06$ & A2007 & 134 & 5.89 \\
\hline 4702.99 & $\mathrm{Mg}_{\mathrm{I}}$ & 4.34 & $3.020 \mathrm{e}-01$ & A2007 & 118 & 6.26 \\
\hline 4102.94 & Si I & 1.91 & $7.943 \mathrm{e}-04$ & MCW & 115 & 5.95 \\
\hline 6155.14 & $\mathrm{Si}$ I & 5.62 & $1.698 \mathrm{e}-01$ & E93 & 23 & 6.07 \\
\hline 6102.73 & $\mathrm{Ca}$ & 1.88 & $1.621 \mathrm{e}-01$ & D2002 & 93 & 4.67 \\
\hline 6122.23 & $\mathrm{Ca}$ I & 1.89 & $4.786 \mathrm{e}-01$ & D2002 & 124 & 4.73 \\
\hline 6161.30 & $\mathrm{Ca}$ & 2.52 & $5.370 \mathrm{e}-02$ & E93 & 22 & 4.75 \\
\hline 6162.18 & $\mathrm{Ca}_{\mathrm{I}}$ & 1.90 & $8.128 \mathrm{e}-01$ & D2002 & 141 & 4.78 \\
\hline 6166.44 & $\mathrm{Ca}$ & 2.52 & $7.244 \mathrm{e}-02$ & R03 & 24 & 4.67 \\
\hline 6169.04 & $\mathrm{Ca}$ I & 2.52 & $1.585 \mathrm{e}-01$ & R03 & 49 & 4.79 \\
\hline 6169.56 & Ca I & 2.53 & $3.311 \mathrm{e}-01$ & DS91 & 56 & 4.60 \\
\hline 6439.08 & $\mathrm{Ca}$ I & 2.52 & $2.951 \mathrm{e}+00$ & D2002 & 117 & 4.62 \\
\hline 6455.60 & Ca I & 2.51 & $5.129 \mathrm{e}-02$ & R03 & 32 & 4.95 \\
\hline 6471.66 & $\mathrm{Ca}$ I & 2.51 & $2.041 \mathrm{e}-01$ & S86 & 46 & 4.60 \\
\hline 6493.79 & $\mathrm{Ca}$ I & 2.52 & $7.762 \mathrm{e}-01$ & DS91 & 92 & 4.76 \\
\hline 5239.82 & $\mathrm{Sc}_{\text {II }}$ & 1.45 & $1.698 \mathrm{e}-01$ & MFW & 66 & 1.36 \\
\hline 5526.82 & Sc II & 1.77 & $1.318 \mathrm{e}+00$ & MFW & 79 & 1.06 \\
\hline 5657.88 & $\mathrm{Sc}$ II & 1.51 & $2.884 \mathrm{e}-01$ & GS & 64 & 1.10 \\
\hline 5669.04 & Sc ${ }_{I I}$ & 1.50 & $7.586 \mathrm{e}-01$ & GS & 66 & 1.70 \\
\hline 6245.62 & $\mathrm{Sc}_{\text {II }}$ & 1.51 & $9.555 \mathrm{e}-01$ & $\mathrm{R} 03$ & 67 & 1.56 \\
\hline 6604.60 & Sc II & 1.36 & $5.012 \mathrm{e}-02$ & R03 & 34 & 1.09 \\
\hline 4533.25 & Ti I & 0.85 & $3.388 \mathrm{e}+00$ & D2002 & 104 & 2.89 \\
\hline 4534.78 & Ti I & 0.84 & $1.905 \mathrm{e}+00$ & D2002 & 95 & 2.96 \\
\hline 4981.72 & Ti I & 0.84 & $3.162 \mathrm{e}+00$ & D2002 & 118 & 2.95 \\
\hline 5866.46 & Ti I & 1.07 & $1.345 \mathrm{e}-01$ & E93 & 31 & 3.16 \\
\hline 6126.22 & Ti I & 1.05 & $4.266 \mathrm{e}-02$ & $\mathrm{R} 03$ & 17 & 3.28 \\
\hline 6261.10 & Ti I & 1.43 & $3.311 \mathrm{e}-01$ & B86 & 28 & 3.16 \\
\hline 5296.70 & $\mathrm{Cr}$ & 0.98 & $4.074 \mathrm{e}-02$ & GS & 76 & 3.56 \\
\hline 5300.75 & Cri & 0.98 & $7.413 \mathrm{e}-03$ & GS & 39 & 3.73 \\
\hline 5345.81 & Cri & 1.00 & $1.047 \mathrm{e}-01$ & GS & 95 & 3.47 \\
\hline 5409.80 & Cri & 1.03 & $1.905 \mathrm{e}-01$ & GS & 109 & 3.46 \\
\hline 6330.09 & Cri & 0.94 & $1.202 \mathrm{e} 093$ & R03 & 18 & 3.91 \\
\hline 5084.11 & $\mathrm{Ni}$ I & 3.680 & $6.607 \mathrm{e}-01$ & E93 & 42 & 4.42 \\
\hline 5115.40 & $\mathrm{Ni}$ I & 3.834 & $5.248 \mathrm{e}-01$ & R03 & 27 & 4.40 \\
\hline 6327.60 & $\mathrm{Ni}$ I & 1.68 & $7.709 \mathrm{e}-04$ & MFW & 22 & 4.28 \\
\hline 6767.77 & $\mathrm{Ni}$ I & 1.83 & $6.761 \mathrm{e}-03$ & MFW & 67 & 4.24 \\
\hline 7788.93 & $\mathrm{Ni}$ I & 1.95 & $1.023 \mathrm{e}-02$ & E93 & 55 & 3.96 \\
\hline 4722.16 & $\mathrm{Zn}$ I & 4.01 & $4.074 \mathrm{e}-01$ & BG80 & 50 & 2.91 \\
\hline 4810.53 & $\mathrm{Zn}$ I & 4.06 & $6.761 \mathrm{e}-01$ & BG80 & 47 & 2.67 \\
\hline 4883.68 & $Y_{\text {II }}$ & 1.08 & $1.175 \mathrm{e}+00$ & SN96 & 86 & 0.41 \\
\hline 5087.43 & $\mathrm{Y}_{\mathrm{II}}$ & 1.08 & 6.761e-01 & SN96 & 64 & 0.14 \\
\hline 5200.41 & $\mathrm{Y}_{\text {II }}$ & 0.99 & $2.692 \mathrm{e}-01$ & SN96 & 52 & 0.20 \\
\hline 5205.72 & $\mathrm{Y}_{\mathrm{II}}$ & 1.03 & $4.571 \mathrm{e}-01$ & SN96 & 62 & 0.19 \\
\hline 6496.90 & Ba II & 0.60 & $4.170 \mathrm{e}-01$ & WM80 & 195 & 1.15 \\
\hline 6320.42 & La II & 0.17 & $3.030 \mathrm{e}-02$ & VWR & 32 & -0.12 \\
\hline 6390.48 & La II & 0.32 & $2.750 \mathrm{e}-02$ & S96 & 42 & 0.14 \\
\hline 6774.33 & La II & 0.12 & $1.778 \mathrm{e}-02$ & S96 & 33 & -0.02 \\
\hline 4073.47 & $\mathrm{Ce}_{\text {II }}$ & 0.48 & $2.089 \mathrm{e}+00$ & SN96 & 82 & 0.69 \\
\hline 4120.84 & $\mathrm{Ce}_{\text {II }}$ & 0.32 & $5.754 \mathrm{e}-01$ & SN96 & 69 & 0.67 \\
\hline 4486.91 & $\mathrm{Ce}_{\text {II }}$ & 0.30 & $4.365 \mathrm{e}-01$ & SN96 & 67 & 0.57 \\
\hline 4562.37 & $\mathrm{Ce}_{\text {II }}$ & 0.48 & $2.138 \mathrm{e}+00$ & SN96 & 84 & 0.47 \\
\hline 4628.16 & $\mathrm{Ce}_{\text {II }}$ & 0.52 & $1.820 \mathrm{e}+00$ & SN96 & 84 & 0.56 \\
\hline 5274.24 & $\mathrm{Ce}_{\text {II }}$ & 1.28 & $2.450 \mathrm{e}+00$ & VWR & 42 & 0.43 \\
\hline 6051.80 & $\mathrm{Ce}_{\text {II }}$ & 0.23 & $2.512 \mathrm{e}-02$ & S96 & 16 & 0.40 \\
\hline 4811.34 & $\mathrm{Nd}$ II & 0.06 & $9.660 \mathrm{e}-02$ & VWR & 66 & 0.55 \\
\hline 4959.12 & $\mathrm{Nd}_{\text {II }}$ & 0.06 & $1.213 \mathrm{e}-01$ & VWR & 73 & 0.56 \\
\hline 4989.95 & $\mathrm{Nd}_{\text {II }}$ & 0.63 & $2.377 \mathrm{e}-01$ & VWR & 49 & 0.55 \\
\hline
\end{tabular}


Table 3. continued.

\begin{tabular}{ccccccc}
\hline \hline$\lambda(\AA)$ & Element & $\chi(\mathrm{eV})$ & $\log g f$ & Ref. & $E W_{\lambda}(\mathrm{m} \AA)$ & $\log \varepsilon$ \\
\hline 5063.72 & $\mathrm{Nd}_{\text {II }}$ & 0.98 & $1.746 \mathrm{e}-01$ & VWR & 27 & 0.69 \\
5089.83 & $\mathrm{Nd}_{\text {II }}$ & 0.20 & $7.244 \mathrm{E}-02$ & $\mathrm{E93}$ & 36 & 0.25 \\
5130.59 & $\mathrm{Nd}_{\text {II }}$ & 1.30 & $1.259 \mathrm{e}+00$ & SN96 & 50 & 0.69 \\
5234.19 & $\mathrm{Nd}_{\text {II }}$ & 0.55 & $3.467 \mathrm{E}-01$ & SN96 & 62 & 0.47 \\
5249.58 & $\mathrm{Nd}_{\text {II }}$ & 0.98 & $1.202 \mathrm{e}+00$ & SN96 & 60 & 0.45 \\
5293.16 & $\mathrm{Nd}_{\text {II }}$ & 0.82 & $6.310 \mathrm{e}-01$ & SN96 & 66 & 0.62 \\
5311.46 & $\mathrm{Nd}_{\text {II }}$ & 0.99 & $2.754 \mathrm{e}-01$ & SN96 & 27 & 0.47 \\
5361.47 & $\mathrm{Nd}_{\text {II }}$ & 0.68 & $3.981 \mathrm{e}-01$ & SN96 & 50 & 0.35 \\
5431.54 & $\mathrm{Nd}_{\text {II }}$ & 1.12 & $3.491 \mathrm{e}-01$ & VWR & 19 & 0.33 \\
5442.26 & $\mathrm{Nd}_{\text {II }}$ & 0.68 & $1.259 \mathrm{e}-01$ & SN96 & 28 & 0.43 \\
5740.88 & $\mathrm{Nd}_{\text {II }}$ & 1.16 & $2.754 \mathrm{e}-01$ & VWR & 23 & 0.56 \\
\hline
\end{tabular}

References. A2007: Aoki et al. (2007); BG80: Biémont \& Godefroid (1980); B81: Biémont et al. (1981); D2002: Depagne et al. (2002); DS91: Drake \& Smith (1991); E93: Edvardsson et al. (1993); GS: Gratton \& Sneden (1988); H82: Hannaford et al. (1982); MFW: Martin et al. (1988); MCW: McWilliam et al. (1995); PS: Preston \& Sneden (2001); R03: Reddy et al. (2003); R04: Reyniers et al. (2004); S86: Smith et al. (1986); S96: Smith et al. (1996); SN96: Sneden et al. (1996); VWR: van Winckel \& Reyniers (2000); WSM: Wiese et al. (1969); WM80: Wiese \& Martin (1980). 